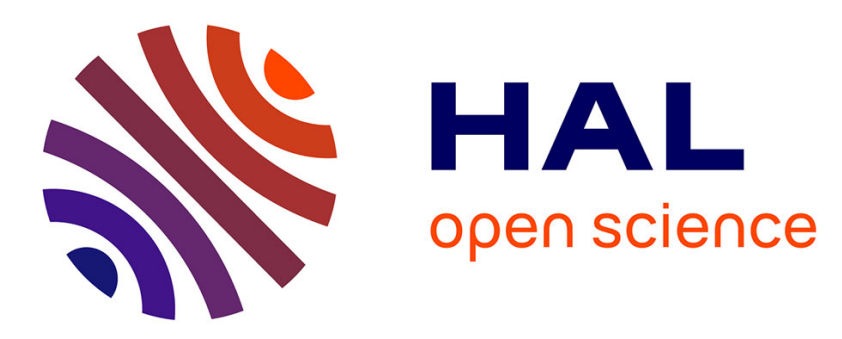

\title{
A new electrical circuit with negative capacitances to enhance resistive shunt damping
}

\author{
Marta Berardengo, Stefano Manzoni, Olivier Thomas, Christophe
}

Giraud-Audine

\section{- To cite this version:}

Marta Berardengo, Stefano Manzoni, Olivier Thomas, Christophe Giraud-Audine. A new electrical circuit with negative capacitances to enhance resistive shunt damping. ASME 2015 Conference on Smart Materials, Adaptive Structures and Intelligent Systems SMASIS 2015, Sep 2015, Colorado Springs, United States. 10.1115/SMASIS2015-8836 . hal-01880312

\section{HAL Id: hal-01880312 \\ https://hal.science/hal-01880312}

Submitted on 24 Sep 2018

HAL is a multi-disciplinary open access archive for the deposit and dissemination of scientific research documents, whether they are published or not. The documents may come from teaching and research institutions in France or abroad, or from public or private research centers.
L'archive ouverte pluridisciplinaire HAL, est destinée au dépôt et à la diffusion de documents scientifiques de niveau recherche, publiés ou non, émanant des établissements d'enseignement et de recherche français ou étrangers, des laboratoires publics ou privés. 


\section{Proceedings of the ASME 2015 Conference on Smart Materials,}

Adaptive Structures and Intelligent Systems

September 21-23, 2015, Colorado Springs, Colorado, USA

\section{SMASIS2015/8836}

\section{A NEW ELECTRICAL CIRCUIT WITH NEGATIVE CAPACITANCES TO ENHANCE RESISTIVE SHUNT DAMPING}

\author{
Marta Berardengo, Stefano Manzoni \\ Politecnico di Milano \\ Department of Mechanics, \\ Via La Masa, 1, 20156 Milano, Italy \\ marta.berardengo@polimi.it \\ stefano.manzoni@polimi.it
}

\author{
Olivier Thomas* \\ Arts et Métiers ParisTech \\ LSIS UMR CNRS 7296 \\ 8 bd. Louis XIV 59046 Lille, France \\ olivier.thomas@ensam.eu
}

\author{
Christophe Giraud-Audine \\ Arts et Métiers ParisTech \\ L2EP \\ 8 bd. Louis XIV 59046 Lille, France \\ christophe.giraud-audine@ensam.eu
}

\begin{abstract}
This article proposes a new layout of electrical network based on two negative capacitance circuits, aimed at increasing the performances of a traditional resistive piezoelectric shunt for structural vibration reduction. It is equivalent to artificially increase the modal electromechanical coupling factor of the electromechanical structure by both decreasing the short-circuit natural frequencies and increasing the open-circuit ones. This leads to higher values of the modal electromechanical coupling factor with respect to simple negative capacitance configurations, when the same margin from stability is considered. This technique is shown to be powerful in enhancing the control performance when associated to a simple resistive shunt, usually avoided because of its poor performances.
\end{abstract}

\section{INTRODUCTION}

Piezoelectric materials are often used in control applications for their ability to convert the mechanical energy of the structure they are bonded on into electrical and conversely. If a proper electrical network is defined and connected to the actuator, it is possible to reduce the vibration level of the structure. This practice is known as piezoelectric shunt damping. The best obtainable performance using an optimized electrical network depends on the characteristics of the Electro Mechanical Structure
(EMS): its intrinsic damping and its modal Electro Mechanical Coupling Factor (MEMCF) [1]. The MEMCF is function of the mechanical, electrical and geometrical characteristics of the piezo-actuator and the structure, for a particular vibration mode. It is also related to the distance between the natural frequencies of the EMS in short (SC) and open circuit (OC) [1,2].

The MEMCF can be maximised to improve the performances by a proper sizing and placement of the piezo-actuator on the structure [3]. Once the piezo-actuator characteristics and its position are optimized and the electrical network is properly tuned, the maximum obtainable performance is fixed. A way to further improve the control performance is to artificially increase the MEMCF. This can be obtained using a Negative Capacitance (NC) [4] connected to the piezo-actuator. A circuit including an operational amplifier is used to synthesize the NC. A NC connected in series is able to decrease the $\mathrm{SC}$ natural frequencies, while a NC in parallel can increase the OC ones [4]. This leads to an increased distance between the $\mathrm{SC}$ and $\mathrm{OC}$ natural frequencies and therefore to a higher MEMCF, which results in better performances. This technique is really effective but suffers of instability problems. The closest the circuit is to the stability limit, the highest the achievable performance is [5]. This paper proposes a new layout of the shunting electrical network based on two NCs, aimed at increasing the MEMCF by both decreasing the $\mathrm{SC}$ and increasing the $\mathrm{OC}$ natural frequencies at the same time. This leads to higher values of MEMCF with respect to simple NC configurations when the same margin from stability

${ }^{*}$ Address all correspondence to this author. 
is considered. This technique is shown to be powerful in enhancing the control performance when associated to a simple resistive shunt, usually avoided because of its poor performances. The present study proposes analytical models to understand the working principle and predict the theoretical tuning. Those theoretical results are validated by experimental tests on a cantilever beam.

\section{ELECTRO-MECHANICAL MODEL Governing equations}

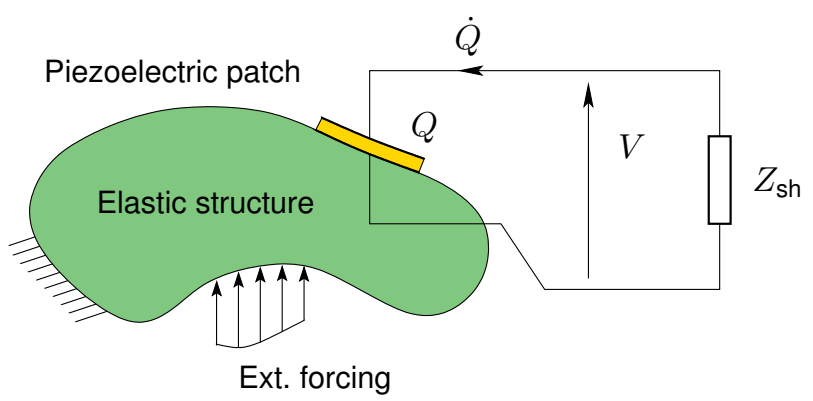

FIGURE 1. A GENERIC STRUCTURE WITH A PIEZOELECTRIC PATCH CONNECTED TO A SHUNT IMPEDANCE $Z_{\text {sh }}$

The model used in this paper to describe the behaviour of the EMS is that presented in the paper of Thomas et al. [1], which was used by its authors to find the optimal tuning of $R$ and $L R$ impedances shunted to a piezoelectric actuator and the associated vibration attenuation performances. We consider a generic elastic structure with one piezoelectric patch, shown in Fig. 1. $U(x, t)$ is the displacement of any point $x$ of the structure at time $t$. An arbitrary shunt impedance $Z_{s h}$ is connected to the piezoelectric patch and $V$ is the voltage between the electrodes of the piezoelectric actuator, which is also the shunt terminal voltage. $Q$ is the electric charge in one of the electrodes, and considering the convention of sign for $V$ in Fig. 1, $Q$ is precisely the charge in the upper electrode. A reduced order model can be obtained by expressing the displacement $U$ in modal coordinates and considering $N$ vibration eigenmodes:

$$
U(x, t)=\sum_{n=1}^{N} \Phi_{i}(x) q_{i}(t)
$$

Where $\Phi_{i}$ is the $i$-th eigenmode of the structure and $q_{i}$ is the $i$-th modal coordinate. The modal coordinates $q_{i}(t)$ are solutions of a problem of the form $[3,6]$ :

$$
\left\{\begin{array}{l}
\ddot{q}_{i}+2 \xi_{i} \omega_{i} \dot{q}_{i}+\omega_{i}^{2} q_{i}-\chi_{i} V=F_{i} \quad \forall i \in 1 \ldots N \\
C_{p} V-Q+\sum_{j=1}^{N} \chi_{j} q_{j}=0
\end{array}\right.
$$

In the above equations, $\omega_{i}$ denotes the $i$-th natural frequency of the EMS in short circuit, $\xi_{i}$ is the $i$-th structural damping factor, $C_{p}$ is the electrical capacitance of the piezoelectric patch and $\chi_{i}$ is the modal coupling coefficient, which represents the energy transfer between the $i$-th mode shape and the piezoelectric patch. Those $\chi_{i}$ coefficients can be computed with either an analytical model [3] or by a finite element model [6]. It must be noted that $\Phi_{i}$ are the eigenmodes of the EMS with the piezo patch in short circuit (SC) (with $V=0$ ) and that $C_{p}$ is the patch capacitance of the blocked structure (with $U(x, t)=0 \forall x \Rightarrow q_{i}=0 \forall i$ ).

The EMS is thus described by $N$ modal equations, corresponding to the balance law of mechanical forces, which describes the equations of motion of the EMS. These equations (2a) are coupled by the term $\chi_{i} V$ to Eq. (2b), which represents the electrical behaviour of the system. Eq. (2b) is related to the balance of electric charges on the piezoelectric electrodes.

According to [1], the following changes of variables can be applied:

$$
\bar{V}=V \sqrt{C_{p}} \quad \bar{Q}=\frac{Q}{\sqrt{C_{p}}}
$$

Substituting Eq. (3) into Eq. (2a) and Eq. (2b), a normalised formulation of Eq. (2a) and Eq. (2b) can be obtained:

$$
\begin{gathered}
\ddot{q}_{i}+2 \xi_{i} \omega_{i} \dot{q}_{i}+\omega_{i}^{2} q_{i}-\omega_{i} k_{i} \sum_{j=1}^{N} \omega_{j} k_{j} q_{j}-\omega_{i} k_{i} \bar{Q}=F_{i} \\
\bar{V}-\bar{Q}+\sum_{j=1}^{N} \omega_{j} k_{j} q_{j}=0
\end{gathered}
$$

where:

$$
k_{i}=\frac{\chi_{i}}{\omega_{i} \sqrt{C_{p}}} .
$$

The above non dimensional parameter $k_{i}$ is defined as the MEMCF. By imposing $\bar{Q}=0$ in Eq. (4a) and reducing the model to a single degree-of-freedom, the following approximated expression of the OC natural frequencies of the EMS is obtained:

$$
\hat{\omega}_{i}=\omega_{i} \sqrt{1+k^{2}}
$$



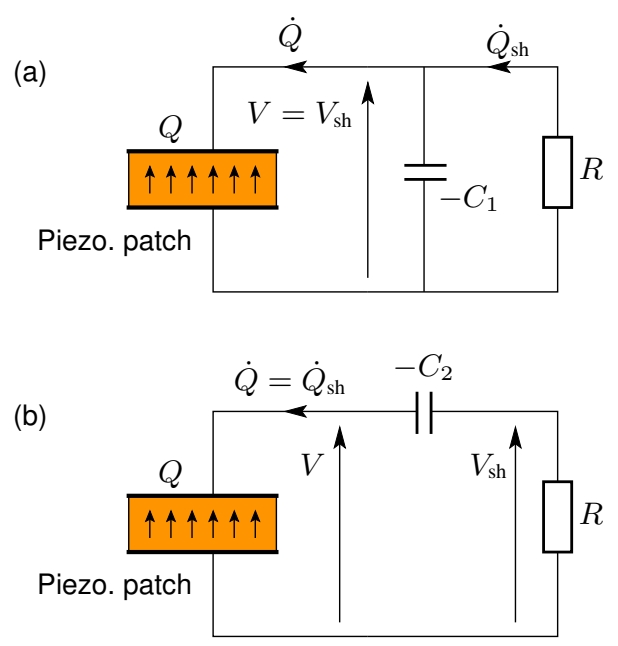

FIGURE 2. RESITIVE SHUNT WITH ADDITION OF A NC: PARALLEL (a) AND SERIES (b) CONFIGURATION

which shows that the MEMCF $k_{i}$ is close to the $i$-th effective coupling factor [1]:

$$
\left|k_{i}\right|=\sqrt{\frac{\left(\omega_{i}^{\mathrm{oc}}\right)^{2}-\left(\omega_{i}^{\mathrm{sc}}\right)^{2}}{\left(\omega_{i}\right)^{2}}} .
$$

where the open and short circuit frequencies are $\omega_{i}^{\text {oc }}=\hat{\omega}_{i}$ and $\omega_{i}^{\mathrm{sc}}=\omega_{i}$.

\section{The EMS with a negative capacitance}

When a NC is used together with a resistive shunt $R$, two shunt circuits can be considered: a parallel configuration (Fig. 2(a)) or a series configuration (Fig. 2(b)). Both schemes are characterised by the presence of an additional negative capacitance $-C_{1}$ or $-C_{2}$. This element is defined by the following relation (Fig. 3(a)):

$$
V_{c}=-\frac{Q_{c}}{C_{n}}
$$

Since a NC does not exist in nature, it is simulated in practice by means of a circuit including an operational amplifier [7]. Such a circuit is depicted in Fig. 3(b). If the operational amplifier is assumed ideal and if its input/output are not saturated, one has:

$$
C_{n}=\hat{C} \frac{R_{2}}{R_{1}}
$$

For the parallel configuration (Fig. 2(a)), the charge $Q$ is the sum of the charges in the negative capacitance $-C_{1}$ branch and (a)

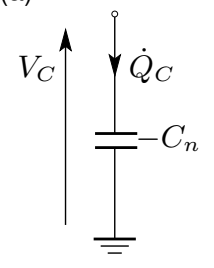

(b)

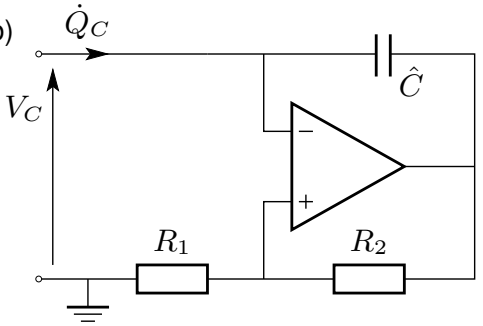

FIGURE 3. DEFINITION OF A NC (a); ELECTRICAL CIRCUIT BASED ON AN OPERATIONAL AMPLIFIER USED TO BUILD A NC TO BE EMPLOYED IN PARALLEL CONFIGURATION (THE PINS OF THE OPERATIONAL AMPLIFIER ARE EXCHANGED FOR THE SERIES CONFIGURATION) (b)

the resistor $R$ branch, so that Eq. ( $2 \mathrm{~b})$ is replaced by:

$$
\left(C_{p}-C_{1}\right) V-Q_{\mathrm{sh}}+\sum_{j=1}^{N} \chi_{j} q_{j}=0
$$

For the series configuration, voltage $V$ is the sum of that at the terminals of the negative capacitance $-C_{2}$ and the shunt resistance $R$, so that Eq. (2b) is replaced by:

$$
V_{\mathrm{sh}}-\left(\frac{1}{C_{p}}-\frac{1}{C_{2}}\right) Q+\sum_{j=1}^{N} \frac{\chi_{j}}{C_{p}} q_{j}=0
$$

In the above equations, $V_{\mathrm{sh}}$ and $Q_{\mathrm{sh}}$ denotes the terminal voltage and the charge that flows into the resistive shunt branch.

In the following, we limit our attention to the response of the $i$-th vibration mode $\left(q_{j}=0 \forall j \neq i\right)$ of the EMS. A full model can be equally considered with a more complex writing of the equations. It is first convenient to define the following equivalent capacitances:

$$
C_{\text {eqp }}=C_{p}-C_{1}, \quad C_{\text {eqs }}=\frac{C_{p} C_{2}}{C_{2}-C_{p}} .
$$

for $C_{p}$ in parallel to $-C_{1}$ and in series to $-C_{2}$ respectively. By applying the same change of variables than Eq. (3):

$$
\bar{V}_{\mathrm{sh}}=V_{\mathrm{sh}} \sqrt{C_{\mathrm{eq}}} \quad \bar{Q}_{\mathrm{sh}}=\frac{Q_{\mathrm{sh}}}{\sqrt{C_{\mathrm{eq}}}}
$$

with $C_{\text {eq }}=C_{\text {eqp }}$ or $C_{\text {eq }}=C_{\text {eqs }}$, the initial problem (2a,b) can be 
TABLE 1. PARAMETERS OF THE EMS ENHANCED BY A SINGLE NEGATIVE CAPACITANCE, IN PARALLEL OR SERIES CONFIGURATION

\begin{tabular}{cccc}
\hline & & Parallel config. & Series config. \\
\hline$\omega_{i}^{\text {sc }}$ & SC freq. & $\omega_{i}$ & $\sqrt{\omega_{i}^{2}-\frac{\chi_{i}^{2}}{C_{2}-C_{p}}}$ \\
\hline$\omega_{i}^{\text {oc }}$ & OC freq. & $\sqrt{\omega_{i}^{2}+\frac{\chi_{i}^{2}}{C_{p}-C_{1}}}$ & $\hat{\omega}_{i}=\sqrt{\omega_{i}^{2}+\frac{\chi_{i}^{2}}{C_{p}}}$ \\
\hline$\tilde{k}_{i}$ & EMEMCF & $\frac{k_{i}}{\sqrt{1-\frac{C_{1}}{C_{p}}}}$ & $\frac{k_{i}}{\sqrt{1-\frac{C_{p}}{C_{2}}}}$ \\
\hline
\end{tabular}

rewritten under the following form:

$$
\left\{\begin{aligned}
\ddot{q}_{i}+2 \xi_{i} \omega_{i} \dot{q}_{i}+\left(\omega_{i}^{\mathrm{sc}}\right)^{2} q_{i}-\omega_{i} \tilde{k}_{i} \bar{V}_{\mathrm{sh}} & =F_{i} \\
\ddot{q}_{i}+2 \xi_{i} \omega_{i} \dot{q}_{i}+\left(\omega_{i}^{\mathrm{oc}}\right)^{2} q_{i}-\omega_{i} \tilde{k}_{i} \bar{Q}_{\mathrm{sh}} & =F_{i} \\
\bar{V}_{\mathrm{sh}}-\bar{Q}_{\mathrm{sh}}+\omega_{i} \tilde{k}_{i} q_{i} & =0
\end{aligned}\right.
$$

The above set of equations defines the dynamics of the EMS viewed from the resistive shunt since the electrical unknowns are now $V_{\mathrm{sh}}$ and $Q_{\mathrm{sh}}$. The following parameters have been defined: $\omega_{i}^{\mathrm{sc}}$ and $\omega_{i}^{\mathrm{oc}}$ are respectively the natural frequency of the EMS with the shunt short-circuited $\left(V_{\mathrm{sh}}=0\right)$ or in open circuit $\left(Q_{\mathrm{sh}}=0\right) . \tilde{k}_{i}$ is the enhanced modal electromecanical coupling factor (EMEMCF), defined as in Eq. (7) with the new values of $\omega_{i}^{\text {sc }}$ and $\omega_{i}^{\text {oc }}$. Those parameters, that take different values in the parallel and series configurations, are gathered in table 1 .

\section{Model with a resistive shunt}

Considering the resistive shunt, $V_{\mathrm{sh}}$ can be expressed as $V_{\text {sh }}=-R \dot{Q}_{\text {sh }}$ so that Eq. (14c) becomes:

$$
\tau_{e} \dot{\bar{Q}}+\bar{Q}_{\mathrm{sh}}-\omega_{i} \tilde{k}_{i} q_{i}=0
$$

with the electrical time constant $\tau_{e}=R C_{\text {eq }}$. Consequently, relying on Eqs. (1), (14b) and (15), the frequency response function (FRF) $H(\Omega)$ between a harmonic force of frequency $\Omega$ applied at a point $x_{f}$ of the mechanical structure and its response at a point $x_{m}$ is:

$$
H(\Omega)=\frac{\Phi_{i}\left(x_{m}\right) \Phi_{i}\left(x_{f}\right)\left(1+j \tau_{e} \Omega\right)}{\left(\omega_{i}^{\mathrm{sc}}\right)^{2}-\left(1+2 \xi_{i} \omega_{i} \tau_{e}\right) \Omega^{2}+j \Omega\left[\tau_{e}\left(\omega_{i}^{\mathrm{oc}}\right)^{2}+2 \xi_{i} \omega_{i}-\tau_{e} \Omega^{2}\right]}
$$

\section{Effect of the Negative Capacitance}

As mentioned in the previous sections and in other works [4], the use of a NC allows to artificially increase the value of the MEMCF and thus to increase the control performances. As introduced in Eqs. (14a-c), it is convenient to analyze the system's behaviour by (i) gathering the EMS and the NC and (ii) by varying the value of the shunt impedance $R$. At its extrema 0 $\left(V_{\mathrm{sh}}=0\right)$ and $+\infty\left(Q_{\mathrm{sh}}=0\right)$, one obtains the natural frequencies of the system in short circuit (SC) $\omega_{i}^{\mathrm{sc}}$ and open circuit (OC) $\omega_{i}^{\mathrm{oc}}$.

In the case of a parallel configuration (Fig. 2(a)), in the SC condition $(R=0)$, the influence of $-C_{1}$ is null since the current is free to flow in the resistor branch. This means that $\omega_{i}^{\mathrm{sc}}=\omega_{i}$ : it does not change with respect to its value without $-C_{1}$. On the contrary, in OC, when $R$ tends to $+\infty$, Tab. 1 shows that the value of $\omega_{i}^{\text {oc }}$ increases with respect to the value of $\hat{\omega}_{i}$ (i.e. without $\left.-C_{1}\right)$.

In the case of the series layout, Fig. 2(b) shows that $\omega_{i}^{\text {oc }}$ does not change with respect to its value without $-C_{2}$ (no current flows in the shunt), while $\omega_{i}^{\text {sc }}$ is decreased with respect to $\omega_{i}$ (i.e. without $-C_{2}$ ).

As a consequence, the effect of the $\mathrm{NC}$ in both configurations is to increase the distance between $\omega_{i}^{\text {sc }}$ and $\omega_{i}^{\text {oc }}$ so that the value of the MEMCF is enhanced, and consequently the attenuation performance of the shunt [1]. The EMEMCF $\tilde{k}_{i}$ (defined by Eq. (7) with the new SC and OC frequencies and given in Tab. 1) is greater than $k_{i}$ and it is a measure of this effect.

\section{Stability}

When a NC is employed, the stability of the EMS coupled to the shunt must be checked because in this case an active component is used. Relying on Eq. (16) and applying the Routh-Hurwitz criterion [8], the following stability conditions are achieved:

$$
\begin{aligned}
& \text { parallel configuration: } \quad C_{1}<C_{p} \\
& \text { series configuration: } \quad C_{2}>C_{p}\left(1+k_{i}^{2}\right)
\end{aligned}
$$

If all the modes of the EMS are taken into account (by using the full model (Eq. (4), see e.g. [4]), condition 17 becomes:

$$
\begin{array}{ll}
\text { parallel configuration: } & C_{1}<C_{\infty} \\
\text { series configuration: } & C_{2}>C_{0}
\end{array}
$$

where

$$
C_{\infty}=C_{p}, \quad C_{0}=C_{p}\left(1+\sum_{i=1}^{N} k_{i}^{2}\right)
$$


$C_{0}$ is the capacitance of the piezoelectric patch at zero frequency $(\Omega=0)$ and $C_{\infty}$ is the capacitance of the piezoelectric patch at infinite frequency $(\Omega \rightarrow+\infty)$. If the EMS is free of external mechanical loading $\left(F_{i}=0 \forall i\right)$, Eqs. $(2(\mathrm{a}, \mathrm{b}))$ enables to compute the piezoelectric patch capacitance $C(\Omega)=Q(\Omega) / V(\Omega)$ as a function of frequency $\Omega$ (it is the time integral of its admittance). One can show (see e.g. [4]) that $C(0)=C_{0}$ as defined above and $C(+\infty)=C_{\infty}=C_{p}$.

The stability limits can also be related to the effects of the negative capacitance on the values of the SC and OC frequencies. In the parallel configuration, if $C_{1} \rightarrow C_{p}$ (see Eq. 17a), $\omega_{i}^{\text {OC }}$ tends to $+\infty$ (see Tab. 1). The destabilization process in this case thus comes from a high frequency mode whose OC frequency reaches $+\infty$. In the series configuration, if $C_{1} \rightarrow C_{0}$ (see Eq. $17 \mathrm{~b}), \omega_{i}^{\mathrm{SC}}$ tends to zero. The destabilization thus comes from a low frequency mode whose SC frequency reaches 0 (see [4] for further details).

\section{NEW NEGATIVE CAPACITANCE LAYOUT}

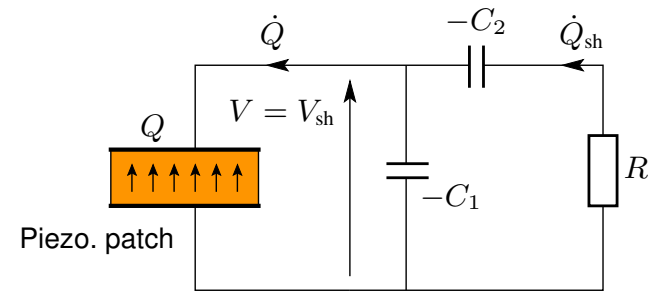

FIGURE 4. EQUIVALENT ELECTRICAL CIRCUIT OF THE NEW SHUNT CONFIGURATION

The previous section explained how the presence of a $\mathrm{NC}$ can increase the EMEMCF and thus the attenuation performance by shifting either $\omega_{i}^{\text {sc }}$ or $\omega_{i}^{\text {oc }}$. The idea of this paper is to use a new shunt circuit able to shift at the same time both $\omega_{i}^{\mathrm{sc}}$ and $\omega_{i}^{\mathrm{oc}}$. This would allow to achieve a further increase of the EMEMCF with respect to the traditional parallel and series configurations. This new circuit is presented in the this section.

\section{Analytical formulation}

The new layout for the shunt impedance relies on the use of two NCs together. This circuit is shown in Fig. 4, where the two NCs are named $-C_{1}$ and $-C_{2}$. Then, a third element is inserted within the circuit, which is the resistance $R$. Therefore, the two NCs are coupled to a resistive shunt and the new layout can be seen as the superimposition of a parallel and a series configuration so that it will be named series+parallel (SP) configuration from here on.
Using the same approach than for the traditional parallel and series configurations, the model of Eqs. 14(a-c) is obtained with the following parameters:

$$
\begin{array}{ll}
\text { equivalent capacitance } & C_{\text {eqsp }}=\frac{\left(C_{p}-C_{1}\right) C_{2}}{C_{1}+C_{2}-C_{p}} \\
\text { SC frequency : } & \omega_{i}^{\text {sc }}=\sqrt{\omega_{i}^{2}-\frac{\chi_{i}^{2}}{C_{1}+C_{2}-C_{p}}} \\
\text { OC frequency : } & \omega_{i}^{\mathrm{oc}}=\sqrt{\omega_{i}^{2}+\frac{\chi_{i}^{2}}{C_{p}-C_{1}}} \\
\text { EMEMCF: } & \tilde{k}_{i}=\frac{k_{i}}{\sqrt{\left(1-\frac{C_{1}}{C_{p}}\right)\left(1+\frac{C_{1}}{C_{2}}-\frac{C_{p}}{C_{2}}\right)}}
\end{array}
$$

When $R$ tends to zero (i.e. SC), the total capacitance of the circuit is $C-C_{1}-C_{2}$ and the value of $\omega_{i}^{\text {sc }}$ is decreased with respect to $\omega_{i}$ (see Eq. (21b) and Fig. 4). Otherwise, if $R$ tends to $+\infty$ (i.e. OC), the total capacitance of the circuit is $C-C_{1}$ and the value of $\omega_{i}^{\text {oc }}$ is increased with respect to $\hat{\omega}_{i}$ (see Eq. (21c)). This means that, as announced, the SP layout allows to change at the same time $\omega_{i}^{\text {sc }}$ or $\omega_{i}^{\text {oc }}$, making their distance greater than in the case of a pure series or parallel configuration. This provides the mentioned increase of $k_{i}$, that is replaced by $\tilde{k}_{i}$ of Eq. (21d).

\section{Stability analysis}

An analogous stability analysis is carried out for the SP configuration by means of the Routh-Hurwitz criterion applied to $H(s)$ (Eq. (16)). The stability conditions are:

$$
C_{1}<C_{p} \quad \text { and } \quad C_{1}+C_{2}>C_{p}\left(1+k_{i}^{2}\right)
$$

Again, if all the modes of the structure are taken into account, the first stability condition becomes $C_{1}<C_{\infty}$ and the second stability condition becomes $C_{1}+C_{2}>C_{0}$, with $C_{0}$ defined by Eq. (20). Again, the same destabilization process hold: if the first condition is not fulfilled, the system is destabilized by a high frequency mode whose OC frequency tends to $+\infty$; if the second condition is not fulfilled, a low frequency mode destabilizes the system with its SC frequency that tends to zero.

\section{Enhanced Modal Electro Mechanical Coupling factor}

The previous sections explained qualitatively why the use of the SP configuration allows to reach a value of the EMEMCF higher than in the case of both the series and parallel layouts. This section will give numerical data about the EMEMCF value 
TABLE 2. DATA OF THE SINGLE-DEGREE-OF-FREEDOM SYSTEMS USED FOR SIMULATIONS

\begin{tabular}{clll}
\hline System & $\omega_{i} /(2 \pi)[\mathrm{Hz}]$ & $\xi_{i}$ & $k_{i}$ \\
\hline 1 & 39.72 & 0.0045 & 0.2716 \\
2 & 986.42 & 0.0028 & 0.1222 \\
\hline
\end{tabular}

which can be achieved using the SP configuration and will show some comparisons with respect to the series and parallel layouts. First a safety margin $\alpha$ is defined, as the distance between the values of NCs and the stability limit, normalised over the value of the stability limit. Hence, considering a single-degreeof-freedom structure and recalling Eq. $(17(a, b)), \alpha$ is defined as:

$$
\begin{array}{ll}
\text { parallel configuration: } & \alpha=\frac{C_{p}-C_{1}}{C_{p}} \\
\text { series configuration: } & \alpha=\frac{C_{2}-C_{p}\left(1+k_{i}^{2}\right)}{C_{p}\left(1+k_{i}^{2}\right)}
\end{array}
$$

As for the SP configuration, two values $\alpha_{1}$ and $\alpha_{2}$ must be defined because two stability conditions (Eq. (22)) must be fulfilled:

$$
\alpha_{1}=\frac{C_{p}-C_{1}}{C_{p}}, \quad \alpha_{2}=\frac{C_{1}+C_{2}-C_{p}\left(1+k_{i}^{2}\right)}{C_{p}\left(1+k_{i}^{2}\right)}
$$

It is noticed that the closer to zero $\alpha, \alpha_{1}$ and $\alpha_{2}$ are, the closer to the stability limit the shunt circuit is. Two different singledegree-of-freedom systems are used here to show the benefits provided by the SP layout in terms of EMEMCF. Their characteristics are shown in Table 2 (these data are chosen because they match with the modal data of two modes of the structure used for experiments; see further in the paper). Fig. 5 shows the value of the EMEMCF, $\tilde{k}_{i}$, achieved with the SP, series and parallel configurations for different values of $\alpha\left(\alpha_{1}=\alpha_{2}=\alpha\right.$ in such a figure) for the two systems considered. Here the capacitance value used is $C_{p}=30.5 \mathrm{nF}$ and will remain the same for all the numerical simulations from here on. The SP layout allows to reach values of $\tilde{k}_{i}$ higher than the series and the parallel with the same safety coefficient, as expected. Hence, the attenuation performance is improved by the SP layout.

\section{OPTIMISATION}

This section aims at explaining how to optimise the values of $C_{1}, C_{2}$ and $R$ (see Fig. 4 ).
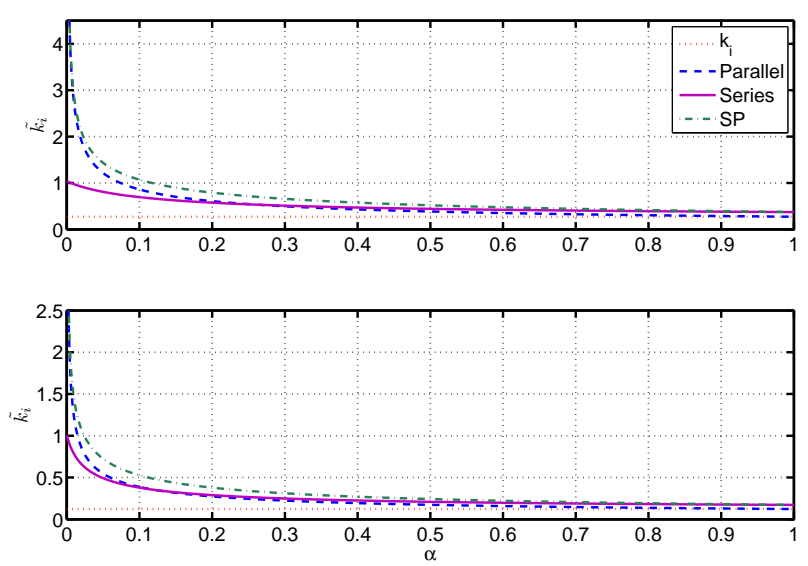

FIGURE 5. TREND OF $\tilde{k}_{i}$ : SYSTEM 1 (UP) AND SYSTEM 2 (DOWN) OF TABLE 2

\section{Optimal Negative Capacitance}

Fig. 5 clearly shows that the closer to the stability limit $C_{1}$ and $C_{2}$ are, the higher the value of $\tilde{k}_{i}$ is, as expected. Therefore, the best performance is achieved with a NC values as close as possible to the stability limit.

\section{Optimal Resistance}

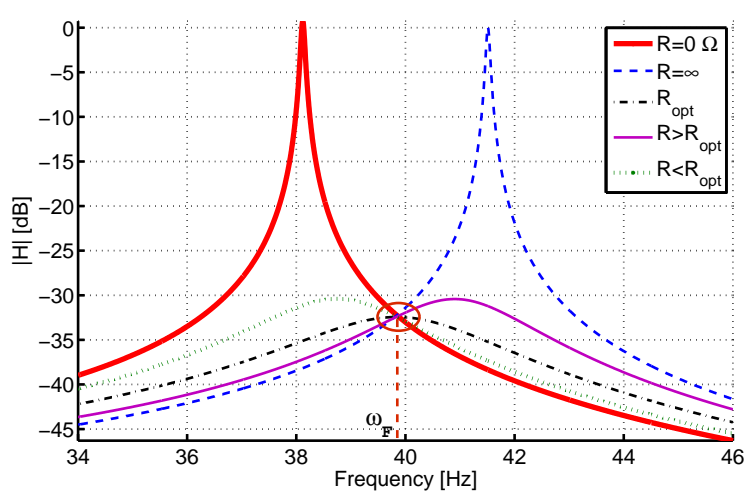

FIGURE 6. TREND OF $|H(\Omega)|$ (WITH SP) FOR DIFFERENT VALUES OF $R$, FROM EQ. (16). THIS SYSTEM HAS $\omega_{i}=2 \pi 39.72$ $\mathrm{rad} / \mathrm{s}, \xi_{i}=0.001, k_{i}=0.2716, C_{1}=6.1 \mathrm{nF}, C_{2}=52.85 \mathrm{nF}$, $R_{\mathrm{opt}}=88.1 \mathrm{k} \Omega$. THE VALUE OF $R<R_{\text {opt }}$ IS $0.5 R_{\text {opt }}$ AND THE VALUE OF $R>R_{\text {opt }}$ IS $2 R_{\text {opt }}$.

Eq.(16) shows that the FRF of the system depends on the value of $R$ through $\tau_{e}$. The optimal value of this last variable can be found by applying the following procedure based on consid- 
erations on the shape of the FRF. As suggested in $[1,2,9,10]$, the optimization problem is solved by neglecting the structural damping $\xi_{i}$. Under such a hypothesis, there exists a point $F$ common to all FRFs $H(\Omega)$ when $R$ is varied (see Fig. 6). The frequency value $\omega_{F}$ associated to point $F$ can be obtained by remarking that among all possible FRFs, this point is common to two particular ones: the one in SC $(R=0)$ (denoted as $H_{0}(\Omega)$ ) and the one in OC $(R \rightarrow+\infty)$ (denoted as $H_{\infty}(\Omega)$ ), for which $H(\omega)$ assumes simple mathematical forms. The frequency $\omega_{F}$ where $H_{\infty}(\Omega)$ and $H_{0}(\Omega)$ cross each other has the following form:

$$
\begin{aligned}
\omega_{F} & =\sqrt{\frac{\left(\omega_{i}^{\mathrm{sc}}\right)^{2}+\left(\omega_{i}^{\mathrm{oc}}\right)^{2}}{2}} \\
& =\omega_{i} \sqrt{1+\frac{k_{i}^{2} C_{p}}{2\left(C_{p}-C_{1}\right)}\left(1-\frac{C_{p}-C_{1}}{C_{2}+C_{1}-C_{p}}\right)}
\end{aligned}
$$

Then, since $F$ is common to all gain curves, the optimum one (the one which has the lowest peak amplitude) has its peak at point $F$. The associated optimal value for $R$ (called $R_{\text {opt }}$ ) can be calculated by solving the following equation as a function of $R$ :

$$
\left.\frac{\partial|H(\Omega)|^{2}}{\partial \Omega}\right|_{\Omega=\omega_{F}}=0
$$

Finally, the expression of $R_{\mathrm{opt}}$ is:

$$
R_{\mathrm{opt}}=\frac{1}{\omega_{F} C_{\mathrm{eqsp}}}=\frac{1}{\omega_{F}}\left(\frac{1}{C-C_{1}}-\frac{1}{C_{2}}\right)
$$

Fig. 7 (which refers to the first system of Table 2) shows that the SP configuration tuned with the optimal value $R_{\mathrm{opt}}$ is able to provide a higher vibration attenuation if compared to the parallel and series layouts (the optimal resistance values for these two configurations were computed numerically) when $\alpha=\alpha_{1}=\alpha_{2}$ (thus with the same safety margin values), as expected.

\section{EXPERIMENTAL RESULTS Test set-up description}

The analytical and numerical results shown previously were validated by a series of experiments. The test set-up was an aluminium cantilever beam (length of $178.8 \mathrm{~mm}$, width of $3.05 \mathrm{~mm}$ and thickness of $1.1 \mathrm{~mm}$ ) with two piezoelectric patches bonded as depicted in Fig. 8. The modes considered for the tests were the first four flexural modes, whose characteristics are given in Table 3 (the shapes of these modes are the usual ones for cantilever beams). The two piezoelectric patches were electrically linked

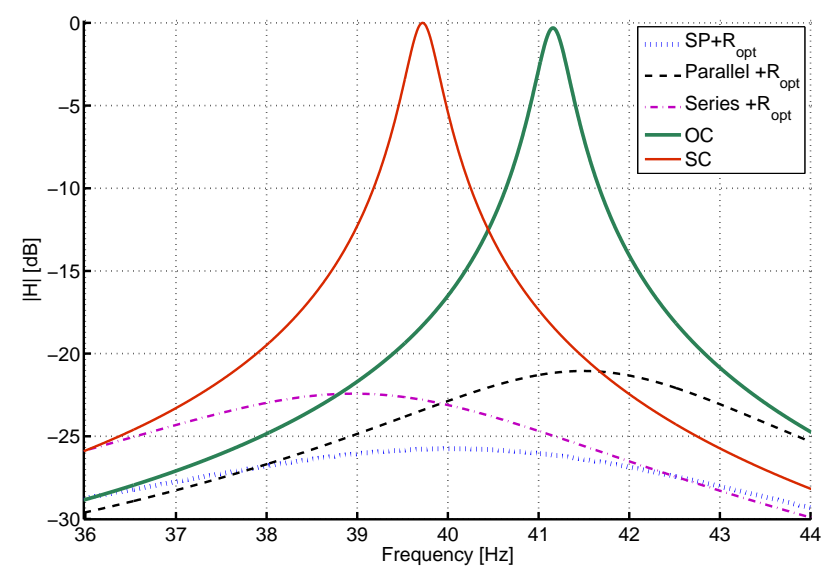

FIGURE 7. $|H|$ FOR THE SC AND OC (WITHOUT NC; SEE THE CURVES NAMED OC AND SC) AND WITH SERIES, PARALLEL AND SP CONFIGURATIONS WITH THE OPTIMAL RESISTANCE. HERE, $\alpha=\alpha_{1}=\alpha_{2}=0.4$. THE FRFS HAVE BEEN DERIVED USING THE THEORETICAL MODEL OF EQ.(16)

TABLE 3. MODAL DATA OF THE EXPERIMENTAL SET-UP

\begin{tabular}{clll}
\hline Mode & $\omega_{i} /(2 \pi)[\mathrm{Hz}]$ & $\xi_{i}$ & $k_{i}$ \\
\hline 1 & 39.72 & 0.0045 & 0.2716 \\
2 & 187.34 & 0.0030 & 0.1037 \\
3 & 509.00 & 0.0026 & 0.0710 \\
4 & 986.42 & 0.0028 & 0.1222 \\
\hline
\end{tabular}

in series and the capacitance value associated to this series is $C_{p}$, estimated as $30.5 \mathrm{nF}$ (a mean value was used averaging the values of $C_{p}$ measured with a multimeter at different frequency values; indeed, the $C_{p}$ values for the four modes were very close to each other). Table 3 shows that the value of $k_{i}$ is high for the first mode and lower for the other three modes. The cantilever beam was excited by a contactless electro-magnetic actuator [11]. The use of such an actuator required to bond a magnet on the beam, close to its tip (see Fig. 8). The vibrational response of the beam was measured by a laser Doppler velocimeter. Finally, the NCs were built with the scheme proposed in Fig. 3. Particularly, $C_{1}$ was built as a parallel NC (Fig. 3) while $C_{2}$ as a series NC (i.e. with the pins of the operational amplifier exchanged with respect to the configuration of Fig. 3). The operational amplifiers employed were OPA445. 


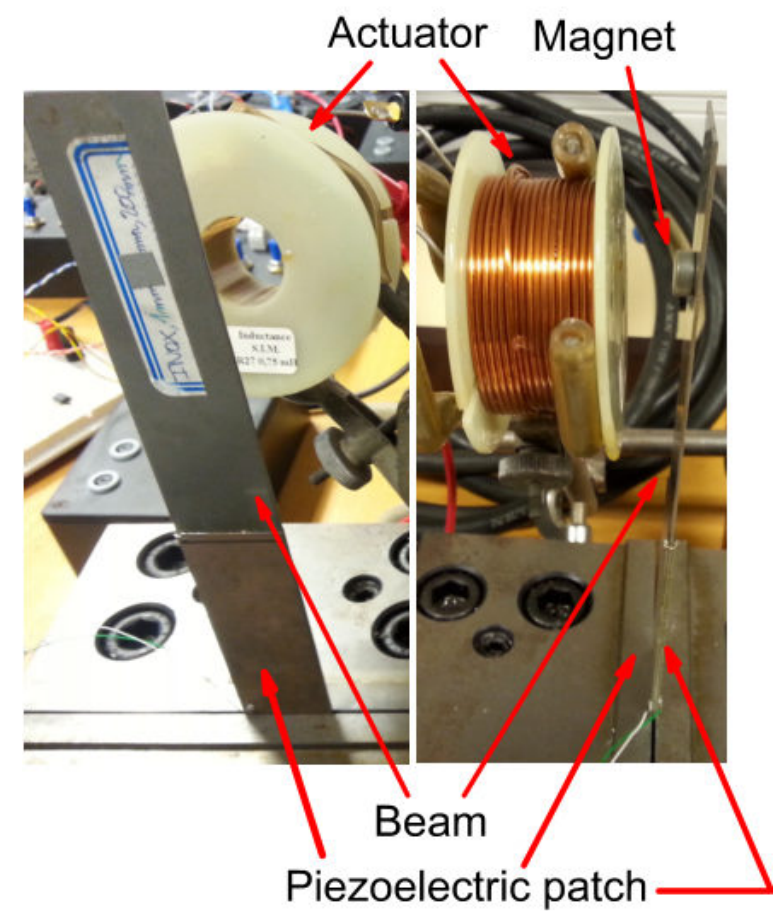

FIGURE 8. EXPERIMENTAL SET-UP

\section{Experimental validation}

The SP circuit was built with different values of $C_{1}, C_{2}$ and $R$ and the resulting FRFs were compared to the analytical expectations. In all the cases the FRFs are expressed in decibels (where the reference is the maximum of the FRF in SC without NCs for the mode considered) in order to have a quick understanding of the attenuation performances. Fig. 9 shows the FRFs for the first mode of Table 3 for the piezoelectric actuator shunted to a SP circuit where three different values for $R$ were chosen: $0 \Omega$ (i.e. the new SC), $+\infty$ (i.e. the new OC) and the optimal value of $R$ fixed by means of Eq. (27). In this test case, $C_{1}=19.825 \mathrm{nF}$, $C_{2}=29.954 \mathrm{nF}$ and $R_{\mathrm{opt}}=237.24 \mathrm{k} \Omega$. The figure shows that the maximum of the FRF with $R_{\mathrm{opt}}$ is close to the intersection point of the two further FRFs related to the SP configuration (i.e. point $F$ ), which is in accordance with the procedure described previously to find the value of $R_{\mathrm{opt}}$. The three FRFs do not cross each other precisely in the same point due to the non-zero mechanical damping $\xi_{i}=0.0045$, as explained in [1]. Furthermore, Fig. 9 also shows that the SP configuration is able to shift both the $\mathrm{SC}$ and the OC eigenfrequencies at the same time, as described previously. Fig. 10 and 11 show two comparisons between experimental and analytical FRFs for the first and fourth modes of Table 3 respectively, when using $R_{\text {opt }}$. The values of the electrical components used for the SP layout for the first mode were $C_{1}=19.825 \mathrm{nF}, C_{2}=17.837 \mathrm{nF}$ and $R_{\mathrm{opt}}=157.89 \mathrm{k} \Omega$, while for the fourth mode $R_{\mathrm{opt}}=6.212 \mathrm{k} \Omega$ (the values of $C_{1}$ and $C_{2}$ are the same). For the series configuration, the negative capacitance value was equal to $C_{1}+C_{2}$. For the parallel configuration, the negative capacitance value was equal to $C_{1}$. The agreement between analytical results (dashed lines) and experiments (solid lines) is very good, proving the reliability of the theoretical approach described previously. The SP configuration shows better performances with respect to the traditional configurations (see Fig. 10 and Fig. 11). The attenuation provided by the SP on the first mode increases of about $3 \mathrm{~dB}$ compared to the series and of about $8 \mathrm{~dB}$ compared to the parallel. As for the fourth mode, the improvement achieved with the SP is of about 2.5 and $8 \mathrm{~dB}$ respectively.

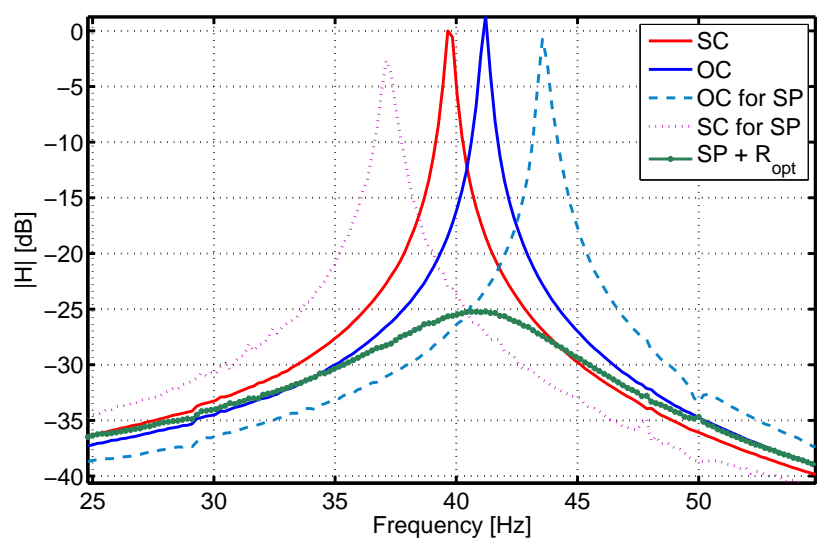

FIGURE 9. $|H|$ FOR THE SC AND OC (WITHOUT NC; SEE THE CURVES NAMED OC AND SC) AND $|H|$ FOR DIFFERENT VALUES OF $R$ (EXPERIMENTAL).

\section{CONCLUSION}

This paper dealt with vibration damping by means of piezoelectric patches shunted to electric impedances based on negative capacitances. The paper proposes a new layout for the shunt impedance, which use the properties of negative capacitances in order to increase the attenuation performance with respect to the traditional way of using the negative capacitance together with resistive shunt. This result is achieved by changing at the same time the short and open circuit resonance frequency of the electromechanical system by designing an impedance where two negative capacitances are present at the same time. Analytical expressions are derived in order to optimally tune the electric elements of the shunt impedance. Furthermore, the numerical simulations confirm that the use of this new impedance allows to increase the modal electromechanical coupling factor with respect to the traditional layouts and thus the vibration attenuation. 


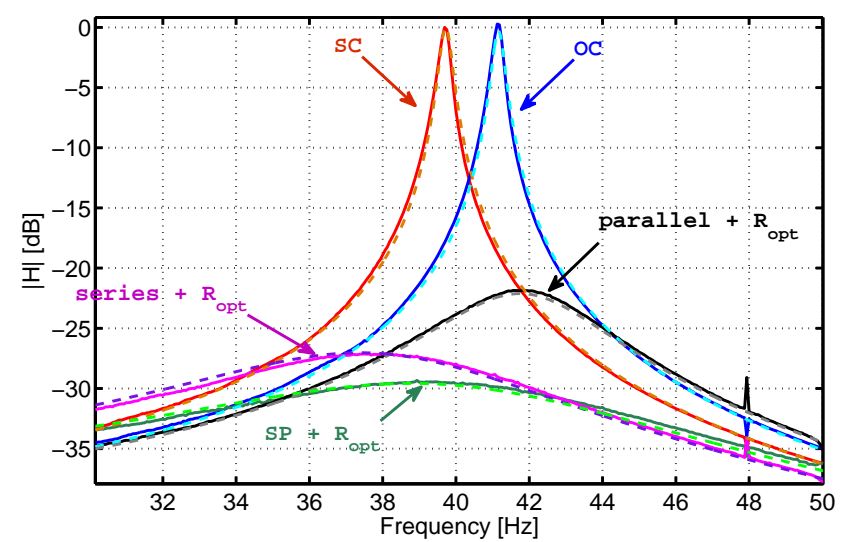

FIGURE 10. $|H|$ FOR THE SC AND OC (WITHOUT NC; SEE THE CURVES NAMED OC AND SC) AND WITH SERIES AND PARALLEL NC WITH THE OPTIMAL RESISTANCE, AND $|H|$ WITH SP AND WITH $R_{\text {opt }}$ FOR THE FIRST MODE OF TABLE 3. THE NUMERICAL (DASHED LINES) AND EXPERIMENTAL (SOLID LINES) CURVES ARE ALMOST SUPERIMPOSED.

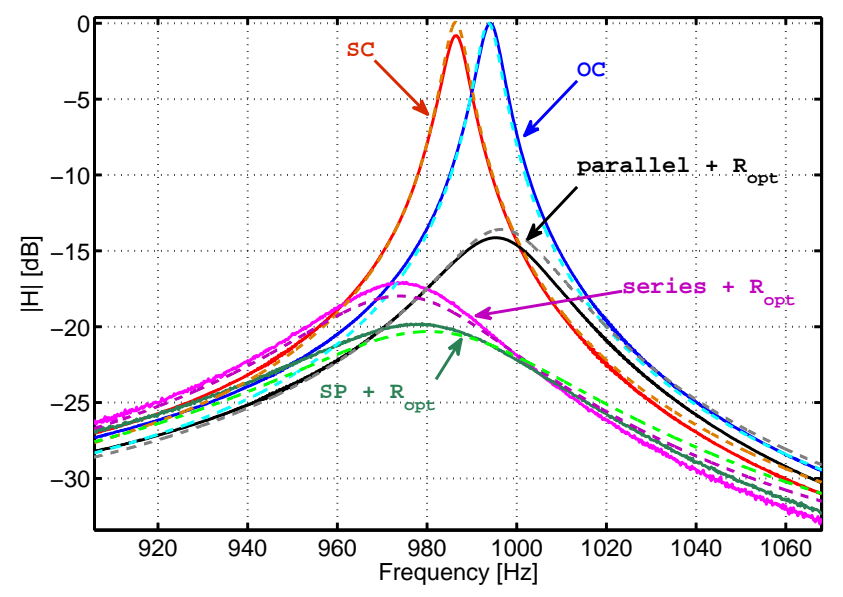

FIGURE 11. $|H|$ FOR THE SC AND OC (WITHOUT NC; SEE THE CURVES NAMED OC AND SC) AND WITH SERIES AND PARALLEL NC WITH THE OPTIMAL RESISTANCE, AND $|H|$ WITH SP AND WITH $R_{\text {opt }}$ FOR THE FOURTH MODE OF TABLE 3. THE NUMERICAL (DASHED LINES) AND EXPERIMENTAL (SOLID LINES) CURVES ARE ALMOST SUPERIMPOSED.

Experiments with a system composed of a cantilever beam and a couple of piezoelectric patches electrically connected in series showed a very good agreement with the analytical and numerical expectations.

\section{REFERENCES}

[1] Thomas, O., Ducarne, J., and Deü, J. F., 2012. "Performance of piezoelectric shunts for vibration reduction". Smart Materials and Structures, 21(1), p. 015008.

[2] Hagood, N., and von Flotow, A., 1991. "Damping of structural vibrations with piezoelectric materials and passive electrical networks". Journal of Sound and Vibration, 146, pp. 243-268.

[3] Ducarne, J., Thomas, O., and Deü, J. F., 2012. "Placement and dimension optimization of shunted piezoelectric patches for vibration reduction". Journal of Sound and Vibration, 331(14), pp. 3286-3303.

[4] de Marneffe, B., and Preumont, A., 2008. "Vibration damping with negative capacitance shunts: theory and experiment". Smart Materials And Structures, 17(3), pp. 863868.

[5] Behrens, S., Fleming, A. J., and Moheimani, S. O. R., 2003. "A broadband controller for shunt piezoelectric damping of structural vibration". Smart Materials and Structures, 12(1), pp. 18-28.

[6] Thomas, O., Deü, J. F., and Ducarne, J., 2009. "Dynamics of an elastic structure with piezoelectric patches: finiteelement formulation and electromechanical coupling coefficients". International Journal for Numerical Methods in Engineering, 80, pp. 235-268.

[7] Horowitz, P., and Hill, W., 1989. The art of electronics, 2nd. ed. Cambridge university press.

[8] Gopal, M., 2002. Control Systems: Principles and Design, 2 ed. Tata McGraw-Hill Education.

[9] den Hartog, J., 1956. Mechanical Vibrations. McGraw Hill.

[10] Snowdon, J., 1968. Vibration and Shock in Damped Mechanical Systems. Wiley.

[11] Thomas, O., Touzé, C., and Chaigne, A., 2003. "Asymmetric non-linear forced vibrations of free-edge circular plates. part ii: experiments". Journal of Sound and Vibration, 265(5), pp. 1075-1101. 